\title{
Optimization of Renewable Pinene Production from the Conversion of Macroalgae
} Saccharina latissima

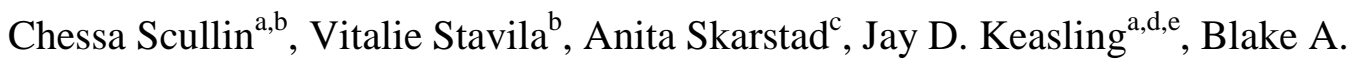 \\ Simmons ${ }^{\mathrm{a}, \mathrm{b}}$, and Seema Singh, ${ }^{\mathrm{a}, \mathrm{b}, *}$ \\ ${ }^{a}$ Joint BioEnergy Institute, Emeryville, CA, USA \\ ${ }^{\mathrm{b}}$ Sandia National Laboratories, Livermore, CA, USA \\ ${ }^{\mathrm{c}}$ Statoil Research Center Trondheim, Trondheim, Norway \\ ${ }^{\mathrm{d}}$ Department of Chemical and Biomolecular Engineering, Department of Bioengineering, \\ University of California, Berkeley, CA 94720, USA \\ ${ }^{e}$ Physical Biosciences Division, Lawrence Berkeley National Laboratory, Berkeley, CA \\ 94720 USA \\ * Corresponding Author
}

Chessa Scullin csscullin@lbl.gov

Vitalie Stavila vnstavi@sandia.gov

Anita Skarstad anisk@statoil.com

Jay D. Keasling jdkeasling@lbl.gov

Blake A. Simmons basimmons @lbl.gov

Seema Singh seesing@sandia.gov

Joint BioEnergy Institute

5885 Hollis Street

Emeryville, CA 94608

Phone: 9252944551

Fax: 5104864252 


\section{Abstract}

Enzymatic hydrolysis of Saccharina latissima with laminarinase was compared to hydrolysis with different combinations of cellulase and hemicellulase enzyme mixtures. The hemicellulase mixture resulted in similar release of glucose, while the cellulase mixture released $40 \%$ more glucose than laminarinase alone. The combination of a laminarinase augmented with a cellulase mixture resulted in a 53\% increase of glucose release from $S$. latissima than laminarinase. Increasing biomass loading above $4 \%(\mathrm{w} / \mathrm{v})$ reduced the sugar yield. Resulting macroalgae hydrolysates were used as a carbon source for the production of pinene, making use of a novel two plasmid Escherichia coli system. The macroalgal hydrolysates were suitable for the novel microbial production of pinene with no further treatment and/or purification.

\section{Keywords}

Macroalgae, Saccharina latissima, renewable pinene, laminarinase, cellulase

\section{Introduction}

Both micro- and macro-algae are thought to minimally compete with established and projected food supplies and estimated to require 4 to 40 times less area than that projected for terrestrial biomass sources to generate significant amounts of renewable chemicals (Adams et al., 2009). Macroalgae has been commercially collected for many years in weights of kilotons in China, Philippines, Indonesia, Chile and numerous countries in Europe with over 16 megatons harvested world wide in 2006, and there are large areas of unused shorelines that have potential for algae cultivation (Roesijad et al., 2010; Vea \& 
Ask, 2011). It has been estimated that using 0.09\% of the potential United States offshore 'Exclusive Economic Zone' to grow macroalgae could replace 1\% of the current United States fuel consumption (Roesijad et al., 2010). Further, countries with current macroalgae farms are developing low impact renewable cultivation methods providing guidance for sustainable farming (Ugarte \& Sharp, 2012; Vea \& Ask, 2011). World-wide commercial production of alginate was valued at 213 million in 2003, with potential for co-production of an easily fermentable sugar source of both mannitol and laminarin (Roesijad et al., 2010). The typical polysaccharide profile of Phaeophyceae (brown algae) includes alginate, fucoidan, mannitol and laminarin. The Phaeophyceae Saccharina latissima has been show to have some of the highest sugar content in the form of laminarin and mannitol (Adams et al., 2009).

Mannitol is synthesized in algae as one of the major photosynthetic products, (Iwamoto \& Shiraiwa, 2005) is found in quantities as high as $20-30 \%$ in brown algae. Laminarin is polysaccharide of $(1,3)-\beta$-D-glucan with $\beta$ - $(1,6)$ branching ending with either a mannitol or a glucose residue approximately $5000 \mathrm{kDa}$ in size (Rioux et al., 2007). It is the primary storage of glucose in the algae, and the focus of fermentation attempts previously with use of a laminarinase (Ross et al., 2011; Ross et al., 2008).

Higher harvesting yields do not necessarily directly equate to higher fermentation yields; rather macroalgal composition plays an important role (Broch \& Slagstad, 2011). The recoverable concentration of fermentable sugars (laminarin, mannitol, and alginate) has been shown to vary with harvest time; a 6 fold reduction in laminarin in S. latissima has been reported to monotonically decrease in harvests from August to December (Broch \& Slagstad, 2011; Holdt \& Kraan, 2011; Peteiro \& Freire, 2009). The regional and seasonal growth differences, even within the same strain, can vastly change the methodology to optimize sugar production for use in biosynthesis. These parameters can impact potential yield and therefor extremely important for estimations of overall feasibility. 
Phaeophyceae have been shown to have promise for biofuel production (ethanol and methanol (Ross et al., 2011; Ross et al., 2008)). Advances in understanding microorganism metabolism have allowed molecular engineering to produce novel and complex biofuels and chemicals, including butanol, fatty acid esters, methyl ethyl ketones and terpenes (Peralta-Yahya et al., 2012). Pinene is a terpene that is a high value chemical precursor for multiple commercial components, such as aromatic chemicals in fragrances, and is currently extracted as a byproduct from the wood pulp industry (Sarria et al., 2014). Components found in hydrolysates of biomass such as salts, phenols, or other materials can inhibit microbial growth and can have an impact on the efficiency of conversion during fermentation. Testing and use of developed microorganisms with biomass-generated hydrolysates is necessary to understand the biomass specific process hurdles that prevent commercialization. In the present work, S. latissima harvested off the Norwegian Coast was characterized and sugar production was evaluated. The hydrolysates were then used to produce pinene with an E. coli-based advanced biosynthesis pathway (Bokinsky et al., 2011; Peralta-Yahya et al., 2012) to demonstrate suitability of hydrolysate resulting from macroalgae.

\section{Materials and Methods}

\subsection{Materials and Preparation}

Samples of Saccharina latissima were collected off the coast of Norway in December 2010, July 2011 and August 2011. The samples were freeze-dried for 1 to 2 days. These samples were stored in $4^{\circ} \mathrm{C}$ before and after shipping. Shipping of the freeze-dried algae to Joint BioEnergy Institute was done at room temperature. Moisture content was continually checked.

\subsection{Total Sugar analysis}

General composition and structural carbohydrates of algae containing mannitol, glucose, xylose, fucose were determined according to the two-step acid hydrolysis procedure of the National Renewable Energy Laboratory (NREL, (Sluiter, 2008)). Carbohydrates were analyzed by high pressure anion exchange chromatography (HPAEC) on an ICS-3000 
system (Dionex, Sunnyvale, CA) equipped with an electrochemical detector and a $4 \mathrm{x}$ $250 \mathrm{~mm}$ CarboPac SA10 analytical column. 1 or $10 \mu \mathrm{L}$ (depending on the concentration) of the sample was injected into the column and was eluted with $1 \mathrm{mM} \mathrm{KOH}$ for $14 \mathrm{~min}$. The flow rate of the eluent was maintained at $1.2 \mathrm{~mL}$ per min. Standards were made for mannitol, glucose, xylose, fucose of 6, 10, 25, 50, and $100 \mu \mathrm{M}$.

\subsection{Enzymatic Saccharification}

Enzymatic saccharification of algae samples was carried out at $50{ }^{\circ} \mathrm{C}$ and $150 \mathrm{rpm}$ in a reciprocating shaker (Enviro-Genie, Scientific Industries, Inc.) in $50 \mathrm{mM}$ citrate buffer (pH of 4.8). The glucan content in the solution was maintained at $5 \mathrm{~g}$ glucan per liter, unless otherwise noted. $20 \mathrm{mg}$ protein per g glucan of Cellic ${ }^{\circledR}$ CTec 2 (Novozymes) and $2 \mathrm{mg}$ protein per g glucan of Cellic® HTec2 (Novozymes) and/or $0.1 \mathrm{U}$ per 20 g glucan laminarinase (1 U per $31 \mathrm{mg}$ Sigma, L5272), (Adams et al., 2009), were used for hydrolysis reactions unless otherwise noted. A $60 \mu \mathrm{L}$ of the supernatant was taken at specific time intervals $(0,0.5,1,2,5,24,48,72 \mathrm{~h})$ to monitor the hydrolysis reaction. The reducing sugars in the supernatant were measured using the 3,5-dinitrosalicylic acid assay, (DNS) or HPAEC.

Solutions of D-glucose were used as standards in the DNS and Amplex assays. All assays were performed with three replicates. Error bars show the standard error of three replicate measurements.

Each saccharification comparison was run concurrently with all samples in the same comparison to eliminate potential differences in temperature history or other parameters. The rate of hydrolysis was calculated based on the sugar released in the first $30 \mathrm{~min}$ of hydrolysis. The supernatant collected after $72 \mathrm{~h}$ of hydrolysis was analyzed with HPAEC for the monosaccharide composition. All assays were performed with three replicates, unless noted. It should be noted that the DNS assay does not account for the hydrolysis reaction stoichiometry of cellulose and hemicellulose upon complete hydrolysis. 


\subsection{Composition}

Ash content was measured using the procedure of NREL (2008). Protein was extracted from algae using glass bead homogenization followed by quantitation with Coomassie Blue (BioRad) with BSA standards. Alginate composition was measured using an adaptation of a multi-step process to get a relative quantifiable amount and was burned to adjust for the ash content of the material (Peña et al., 1997; Rioux et al., 2007) Briefly, alginate was extracted using the multistep process described in (Peña et al., 1997) with $\mathrm{Na}_{2} \mathrm{CO}_{3}$ and $\mathrm{CaCl}_{2}$. The recovered alginate was then ashed at $575^{\circ} \mathrm{C}$ for $3 \mathrm{~h}$ to account for the mineral content, the difference between the $105{ }^{\circ} \mathrm{C}$ dried weight and the ashed values were used to calculated the alginate concentration. Conductivity was measured using a pocket conductivity meter (B-173, Horiba, Edison, NJ), samples were diluted 10 and 100 fold and confirmed for linearity of measurement.

\subsection{Confocal Fluorescence Imaging}

S. latissima samples were cut with a razor blade from freeze-dried samples from the December 2010 harvest. These sections were stored at $4^{\circ} \mathrm{C}$ until imaged. Slices were placed between a coverslip and slide with $150 \mu \mathrm{L}$ buffered enzyme to wet each sample. Autofluorescence images during heating were collected with a Zeiss LSM 710 confocal system mounted on a Zeiss inverted microscope (Carl Zeiss Microscopy, LLC, Thornwood, $\mathrm{NY}$ ). A $405 \mathrm{~nm}$ diode laser and a $488 \mathrm{~nm}$ argon laser were used for sequential excitation over a 410-759 $\mathrm{nm}$ range with $5 \mathrm{x}, 10 \mathrm{x}$ or $40 \mathrm{x}$ objectives. The resulting images were analyzed using the Zen software and reproduced in pseudo color (Zeiss).

\subsection{Pinene production in E. coli MG1655.}

E. coli was co-transformed with pJBEI-3085 carrying the mevalonate pathway from aceoacetyl-CoA transferase to isopentenyl diphosphate isomerase (Bokinsky et al., 2011) and JBEI - 3933 carrying a protein fusions of Abies grandis pinene synthase linked to A. grandis geranyl diphospahte synthase using a (Gly-Ser-Gly) $)_{2}$ linker (Sarria et al., 2014). Pre-cultures of E. coli MG1655 harboring the appropriate plasmids were used to inoculate at a 1:25 dilution pinene production medium (Teknova, EZ-Rich, 1\% (v/v) glucose, 100mg 
per $\mathrm{L}$ ampicillin, $30 \mathrm{mg}$ per $\mathrm{L}$ chloramphenicol, $5 \mathrm{ml}$ total volume). The cultures were grown at $37^{\circ} \mathrm{C}$ for $3 \mathrm{~h}$ ( 200 r.p.m. $\left.\mathrm{OD}_{600}=0.6-0.8\right)$ before induction with $1 \mathrm{mM}$ IPTG and overlayed with $20 \%$ dodecane. After growth for $72 \mathrm{hrs}$ at $30{ }^{\circ} \mathrm{C}(200$ r.p.m. $), 10 \mu \mathrm{L}$ of the dodecane overlay were sampled and diluted into $90 \mu$ of ethyl acetate spiked with terpinene as an internal standard. The samples were analysed by gas chromatography-mass spectrometry (GCMS, Agilent 6890 with Agilent 5973 Mass selective detector) with a DB5 column $(30 \mathrm{~m} \times 0.25 \mathrm{~mm}$ ID $\times 0.25 \mu \mathrm{m}$ film) using either an alpha or beta pinene standard curve using the following conditions: inlet at $230^{\circ} \mathrm{C}, 2 \mathrm{ml} \mathrm{min}^{-1}$ constant flow, transfer line at $300{ }^{\circ} \mathrm{C}$, ion source at $230{ }^{\circ} \mathrm{C}$, scan $\mathrm{m} / \mathrm{z}, 50-300$. Oven: $60{ }^{\circ} \mathrm{C}$ for $1 \mathrm{~min}$, ramp at $20^{\circ} \mathrm{C}$ $\min ^{-1}$ to $120^{\circ} \mathrm{C}$, ramp at $50^{\circ} \mathrm{C}$ to $250^{\circ} \mathrm{C}$.

\section{Results and Discussion}

\subsection{Compositional Characterization}

Analysis of S. latissima harvested off the coast of Norway showed compositional differences between seasonal harvests, as expected. The July and August 2011 harvests contained 11-18\% glucan (laminarin), 17-25\% alginate, 20-23\% mannitol, 30-37\% ash, and $<1 \%$ protein (Table 1). The July and August compositional profiles were similar to the profiles of brown macroalgae harvested at a similar seasonal harvests (17- 34\% alginate, 0$33 \%$ laminarin, 2-19\% mannitol, 0.06 to $15 \%$ protein, 15 to $45 \%$ ash, with concentration of mannitol and laminarin peaking in the summer months) (Adams et al., 2011; Holdt \& Kraan, 2011). Unexpectedly, the December 2010 harvest has similar amounts of glucose as the August harvest, namely 10\% and 13\% respectively (Broch \& Slagstad, 2011; Holdt \& Kraan, 2011; Peteiro \& Freire, 2009). The mannitol content of the December harvest was $6 \%$, which is lower than previously reported, but similar to that previously reported for January. The other compositional components of the December harvest were similar to those reported: $43 \%$ alginate, $25 \%$ ash, and $<1 \%$ protein (Table 1) (Broch \& Slagstad, 2011; Holdt \& Kraan, 2011; Peteiro \& Freire, 2009). While the composition is slightly different from previously reported batches, composition is expected to change with specific species, seasonal variation and harvesting methods. The differences could also be due to 
growth location since the previous published reports on S. latissima composition were not algae harvested off the coast of Norway.

The extracted alginate showed that the mannuronic acid to guluronic acid ratio as measured by the ratio of FTIR peaks at $1030 \mathrm{~cm}^{-1}$ to $1090 \mathrm{~cm}^{-1}$, were $0.64 \pm 0.02,0.64 \pm 0.02$ to $0.686 \pm 0.005$ for July, December and August respectively suggesting while seasonal variations in the amount of alginate minimal differences in overall composition suggesting minimal seasonal difference in viscosity (Chee et al., 2011; Peña et al., 1997).

\subsection{Sugar yields as a function of enzyme mixture}

Enzymatic saccharification of S. latissima with laminarinase (endo - (1,3(4) - B-gluconase)) resulted in a similar sugar release to those reported by Adams et. al (Adams et al., 2009), and is used as a baseline to compare our harvested samples with the published reports of glucose release (Figure 1). To enhance sugar release, we used a commercial cellulase mixture (Cellic ${ }^{\circledR}$ CTec2) and a combination of enzymes optimized for digestion of cellulosic biomass. Use of these commercial enzyme mixtures assisted in additional hydrolysis of $\beta$-(1-4) glucan bonds, resulting in more monomeric glucose release than that obtained with the laminarinase alone (5\% increase with HTec2, 42\% increase with CTec2, $53 \%$ increase with laminarinase and CTec2, and 65\% increase with CTec2 and HTec2, supplemental movie 1, Figure 1). There was further enhancement of glucose release when using the combination of CTec2 with either laminarinase or HTec2, a hemicellulose mixture (Figure 1). In addition, there was a 2-fold increase in the rate of hydrolysis using $\mathrm{CTec} 2$ and additional release with the combination of CTec2 with either HTec2 or laminarinase than either enzyme alone (Figure 1). We also tested the effect of enzyme loading and mechanical grinding of biomass on total sugar release from S. latissima. When the enzyme loading of CTec 2 and HTec 2 was reduced from $20 \mathrm{mg}$ per g glucan to $5 \mathrm{mg}$ per g glucan with a $5 \mathrm{~g}$ glucan per liter loading, no change in yield of final glucose was observed. In addition, there was equivalent sugar released from ground (40 mesh) and unground $\left(\sim 1-2 \mathrm{~cm}^{2}\right)$ algae biomass of S. latissima. The improved kinetics and enhanced glucose release of the reactions supplemented with CTec 2 during enzymatic hydrolysis 
suggests the digestion of a number of $\beta(1,4)$ glucan bonds in macroalgae that had been left undigested in previous studies (Ross et al., 2011; Ross et al., 2008).

Confocal auto-fluorescent images were recorded during enzymatic saccharification to investigate hydrolytic mechanism. As expected from the enzymatic hydrolysis, there is immediate breakdown of the biomass during the first 5 hours (Supplementary Figure 1). This can be seen by the diffusion of cellular fragments away from the algae, (yellow arrows, Supplementary Figure 1). The breakdown of the algae is expected to occur even faster during the batch enzymatic hydrolysis due to mixing effect than shown on the video since algal biomass hydrolysis was performed in static mode on a coverslip for the movie. Interestingly, the outer peripheral layer as seen on the left side of the slice (white arrow, Supplementary Figure 1) seems to mechanistically inhibit biomass degradation. This outer layer has been shown to contain inhibitory chemicals, phenols and tannins, and provides mechanical support (Moen et al., 1997). These results suggest that a more comprehensive pretreatment and hydrolysis steps targeting these components of first removing this layer may enhance the enzyme kinetics.

\subsection{Impact of Increased Biomass Loading on Sugar Yields}

Higher biomass loading during saccharification to generate a suitable sugar stream for downstream fermentation is necessary to make the process more industrially relevant by reducing operating costs. Increased loading from 2,4 , and $8 \%(\mathrm{w} / \mathrm{v})$ biomass $(0.5 \%, 1 \%$, $2 \%$ glucan loading) during saccharification resulted in sugar yields of $10.3 \%, 10.0 \%$, to $3 \%$ gram glucose per gram biomass per liter for the July harvest, respectively. Previous studies have hypothesized a salt sensitivity on saccharification/ethanol yields above $90 \mathrm{mM}$ (Adams et al., 2009; Adams et al., 2011). Multiple sharp peaks in the XRD pattern of the microalgae suggest a high level of sea salt that matches with the compositional analysis. The crystalline peaks present in the XRD pattern were indexed to the following phases: $\mathrm{NaCl}(\mathrm{Fm}-3 \mathrm{~m}), \mathrm{KCl}(\mathrm{Fm}-3 \mathrm{~m}), \mathrm{CaCO}_{3}(\mathrm{Pmcn})$ and D-mannitol (P2 1$)$. Additionally, high ash content supports that high ionic composition could play a role in the inhibition (Table 1). 
To understand the effect of salt inhibition, the algae samples were rinsed using deionized water using a wash volume from $1 \mathrm{~mL}$ per $\mathrm{g}$ algae to $8 \mathrm{~mL}$ per $\mathrm{g}$ algae. This was done quickly as to minimize the absorption into the algae samples. The recovered wash was analyzed for salt (conductivity) and soluble sugars. The lower wash volumes ( $1 \mathrm{~mL}$ per $\mathrm{g}$ algae) had high concentrations of both salt (19 to $32 \mathrm{mS} / \mathrm{cm}$ ) and sugars ( $\mathrm{g} / \mathrm{L})$. Increasing the wash volume ( $8 \mathrm{~mL}$ per $\mathrm{g}$ algae) did not increase the proportional amount of salt ( 8 to $13 \mathrm{mS} / \mathrm{cm})$ to sugar $(0.2 \mathrm{~g} / \mathrm{L})$ lost in the wash. The lowest wash volume (1 mL per $\mathrm{g}$ algae) resulted in $71 \%$ recovery of the initial biomass (dry weight). To confirm the loss of salt and other insoluble material the complete composition of the recovered algae was analyzed (Table 1). Washing increased the alginate percentage of biomass (25 to 39\% alginate for July, and 17.3 to $35 \%$ alginate for August), but reduced the total mass of ash in the samples (30.3 to $28 \%$ ash for July, 37.6 to $33.5 \%$ for August). There was only a minor difference in glucose concentration (17.9 to $17.1 \%$ for July, and 10.2 to $12.6 \%$ for August, Table 1).

The washed samples had reduced recovery compared to the unwashed samples at the same glucan loadings $0.5 \%$ to $2 \%(\mathrm{w} / \mathrm{v})$. At $0.5 \%$ glucan loading the monomeric sugar recovery of the washed sample was $94 \%$ of the glucose recovery from the unwashed sample, and at $1 \%$ glucan loading the washed sample had $50 \%$ of the recovery than the unwashed samples. Washing reduced the glucose recovery to an undetectable amount at loadings above $1 \%$ glucan $(\mathrm{w} / \mathrm{v})$. The lower ratio of glucose to alginate in the total biomass required higher total alginate loading to achieve similar loadings of glucan for the washed material versus the raw biomass. Increased alginate concentration is known to significantly increase viscosity (Chee et al., 2011; Peña et al., 1997). The washed biomass had a higher alginate to glucan ratio than the unwashed samples and therefore increased viscosity, which could be acting as an inhibitor due to uneven mixing. A more intense washing could help reduce the viscosity of the alginate by removing ions such as calcium which is known to help stabilize the alginate and increase the viscosity, however, the more vigorous the wash, the more easily released sugar is dissolved into the wash solution. 
A combination of laminarinase and cellulolytic biomass enzymes increases the glucose release, but rinsing the algae decreases the sugar yields. Rinsing the algae results in loss of mannitol and small easily dissolvable sugars, which requires additional steps to recover. However, rinsing reduces the overall phenol and high salt content during hydrolysis, which if optimized could increase the sugar yield (Adams et al., 2009; Adams et al., 2011).

\subsection{Pinene Production from Macroalgal Hydrolysate}

Previous efforts to produce pinene from other biomass sources has resulted in production around $1 \mathrm{mg}$ per $\mathrm{L}$ for $2 \%$ switchgrass loading, equivalent to a $1 \%$ glucose hydrolysate loading. As scaffolding has been shown to increase flux through metabolic pathways, a protein fusion of pinene synthase and geranyl diphosphate synthase was made to increase the pinene titers (Sarria et al., 2014). Using this E. coli-based production system, pinene was produced from both the hydrolysates obtained from macroalgae and a glucose control. Interestingly, a $0.6 \%(\mathrm{w} / \mathrm{v})$ glucose loading which resulted from $60 \%$ of the medium being replaced with a $1 \%(\mathrm{w} / \mathrm{v})$ glucose hydrolysate resulted in similar pinene production $\sim 20$ $\mathrm{mg} / \mathrm{L}$ versus $18 \mathrm{mg} / \mathrm{L}$, suggesting no effect from inhibitors at this concentration. The hydrolysate made using either the $\mathrm{CTec} 2$ and $\mathrm{HTec} 2$ or $\mathrm{CTec} 2$ and laminarinase contained the largest amount of sugars and produced similar amounts of pinene $\sim 21 \mathrm{mg} / \mathrm{L}$. The unground, but crushed samples, produced similar amount of sugar as the ground samples after saccharification, and the hydrolysates also resulted in similar pinene production $\sim 18$ $\mathrm{mg} / \mathrm{L}$ (Figure 2). Doubling the solids loading for the saccharification step resulted in the expected increase in sugar present in the hydrolysate $(2 \%(\mathrm{w} / \mathrm{v})$ glucose hydrolysate, a 1.2 $\%(w / v)$ final concentration), but the pinene production was significantly reduced to $8 \%$ of the production of the $1 \%(\mathrm{w} / \mathrm{v})$ glucose hydrolysate $(0.6 \%(\mathrm{w} / \mathrm{v})$ final concentration). The reduction in yield could be due to higher sensitivity to the increased viscosity from alginate or the increased salt levels that generate an inhibitory effect similar to that observed during saccharification of the washed samples.

\section{Conclusion}


Macroalgae shows promise as a potential biomass source for production of fermentable sugars and for advanced biochemical production. A mixture of CTec2 and HTec2 releases the maximum amount of glucose from S. latissima. The hydrolysates generated from $S$. latissima are as effective as the glucose control for fermentation with E. coli to make the renewable chemical pinene. Increasing the solids loading to a more relevant industrial concentration is not straightforward due to inhibitory effects of increasing alginate and salt concentration. The specific characteristics of the hydrolysate such as viscosity and salt concentration are important for increasing product yields.

\section{Acknowledgements}

The authors would like to thank Børre Tore Børresen, Evy Mellemsæther and Hans Kristian Kotlar for discussions of this work and Doug Higgins and Jason Lupoi for their reading of the manuscript. This work was supported through a collaborative research grant with Statoil Research Center Trondheim, Trondheim, Norway. The portion of the work conducted by the Joint BioEnergy Institute was supported by the Office of Science, Office of Biological and Environmental Research, of the U.S. Department of Energy under Contract No. DEAC02-05CH11231. The authors thank Novozymes for the gift of the enzyme mixtures (CTec2 and HTec2) used in this work.

\section{References}

1. Adams, J.M., Gallagher, J.A., Donnison, I.S. 2009. Fermentation study on Saccharina latissima for bioethanol production considering variable pre-treatments. Journal of Applied Phycology, 21(5), 569-574.

2. Adams, J.M., Ross, A.B., Anastasakis, K., Hodgson, E.M., Gallagher, J.A., Jones, J.M., Donnison, I.S. 2011. Seasonal variation in the chemical composition of the bioenergy feedstock Laminaria digitata for thermochemical conversion. Bioresource Technology, 102(1), 226-34.

3. Bokinsky, G., Peralta-Yahya, P.P., George, A., Holmes, B.M., Steen, E.J., Dietrich, J., Lee, T.S., Tullman-Ercek, D., Voigt, C.A., Simmons, B.A., Keasling, J.D. 2011. Synthesis of three advanced biofuels from ionic liquid-pretreated switchgrass using engineered Escherichia coli. Proceedings of the National Academy of Sciences of the United States of America, 108(50), 19949-19954.

4. Broch, O., Slagstad, D. 2011. Modelling seasonal growth and composition of the kelp Saccharina latissima. Journal of Applied Phycology, 1-18. 
5. Chee, S.-Y., Wong, P.-K., Wong, C.-L. 2011. Extraction and characterisation of alginate from brown seaweeds (Fucales, Phaeophyceae) collected from Port Dickson, Peninsular Malaysia. Journal of Applied Phycology, 23(2), 191-196.

6. Holdt, S.L., Kraan, S. 2011. Bioactive compounds in seaweed: functional food applications and legislation. Journal of Applied Phycology, 23(3), 543-597.

7. Iwamoto, K., Shiraiwa, Y. 2005. Salt-regulated mannitol metabolism in algae. Marine Biotechnology, 7(5), 407-415.

8. Moen, E., Horn, S., Ostgaard, K. 1997. Alginate degradation during anaerobic digestion of Laminaria hyperborea stipes. Journal of Applied Phycology, 9(2), 157-166.

9. Peña, C., Campos, N., Galindo, E. 1997. Changes in alginate molecular mass distributions, broth viscosity and morphology of \&lt;i\&gt;Azotobacter vinelandii\&lt;/i\&gt; cultured in shake flasks. Applied Microbiology and Biotechnology, 48(4), 510-515.

10. Peralta-Yahya, P.P., Zhang, F., del Cardayre, S.B., Keasling, J.D. 2012. Microbial engineering for the production of advanced biofuels. Nature, 488(7411), 320-8.

11. Peteiro, C., Freire, 0. 2009. Effect of outplanting time on commercial cultivation of kelp Laminaria saccharina at the southern limit in the Atlantic coast, NW Spain. Chinese Journal of Oceanology and Limnology, 27(1), 54-60.

12. Rioux, L.E., Turgeon, S.L., Beaulieu, M. 2007. Characterization of polysaccharides extracted from brown seaweeds. Carbohydrate Polymers, 69(3), 530-537.

13. Roesijad, G., Jones, S.B., Snowden-Swan, L.J., Zhu, Y. 2010. Macroalgae as a biomass feedstock: a preliminary analysis. Pacific Northwest National Laboratory, USA. PNNL 19944.

14. Ross, A.B., Adams, J.M.M., Anastasakis, K., Hodgson, E.M., Gallagher, J.A., Jones, J.M., Donnison, I.S. 2011. Seasonal variation in the chemical composition of the bioenergy feedstock Laminaria digitata for thermochemical conversion. Bioresource Technology, 102(1), 226-234.

15. Ross, A.B., Jones, J.M., Kubacki, M.L., Bridgeman, T. 2008. Classification of macroalgae as fuel and its thermochemical behaviour. Bioresource Technology, 99(14), 6494-6504.

16. Sarria, S., Wong, B., Garcia Martin, H., Keasling, J.D., Peralta-Yahya, P. 2014. Microbial synthesis of pinene. ACS Synth Biol, 3(7), 466-75.

17. Sluiter, A. 2008. Determination of structural carbohydrates and lignin in biomass [electronic resource] : laboratory analytical procedure (LAP) : issue date, 4/25/2008 / A. Sluiter ... [et al.]. National Renewable Energy Laboratory, Golden, Colo. :.

18. Sluiter, A., Hames, B., Ruiz, R., Scarlata, C., Sluiter, J., Templeton, D. 2008. Determination of ash in biomass [electronic resource] : laboratory analytical procedure (LAP) : issue date, 7/17/2005 / A. Sluiter ... [et al.]. National Renewable Energy Laboratory, Golden, Colo. :

19. Ugarte, R., Sharp, G. 2012. Management and production of the brown algae Ascophyllum nodosum in the Canadian maritimes. Journal of Applied Phycology, 24(3), 409-416. 
20. Vea, J., Ask, E. 2011. Creating a sustainable commercial harvest of Laminaria hyperborea, in Norway. Journal of Applied Phycology, 23(3), 489-494.

Figure 1: Enzymatic saccharification of macroalgae. A. Final sugar yields from enzymatic saccharification of macroalgae, $5 \mathrm{~mL}$ at $5 \mathrm{mg}$ glucose per $\mathrm{mL}, 50^{\circ} \mathrm{C} 72$ hours glucose measured by HPAEC. B. Hydrolysis rate obtained as a function of enzyme used, based on DNS assay reducing sugar release at $30 \mathrm{~min}$. Values show with $\pm 1 \mathrm{SD}$.

Figure 2: Pinene production from glucose control $(0,0.3 \%, 0.6 \%, 1 \%, 2 \%(\mathrm{w} / \mathrm{v})$ glucose $)$ and enzymatic saccharification hydrolysates derived from macroalgae enzymatic hydrolysis with CTec2 and HTec2 for December 2010 harvest at $0.6 \%$ glucose loading, and a twice as concentrated hydrolysate (1.2\% glucose loading equal volume replaced). Values shown \pm 1 SD. 


Table 1: Composition of macroalgae samples as a function of seasonal harvest, values
shown at \pm 1 SD.

28 


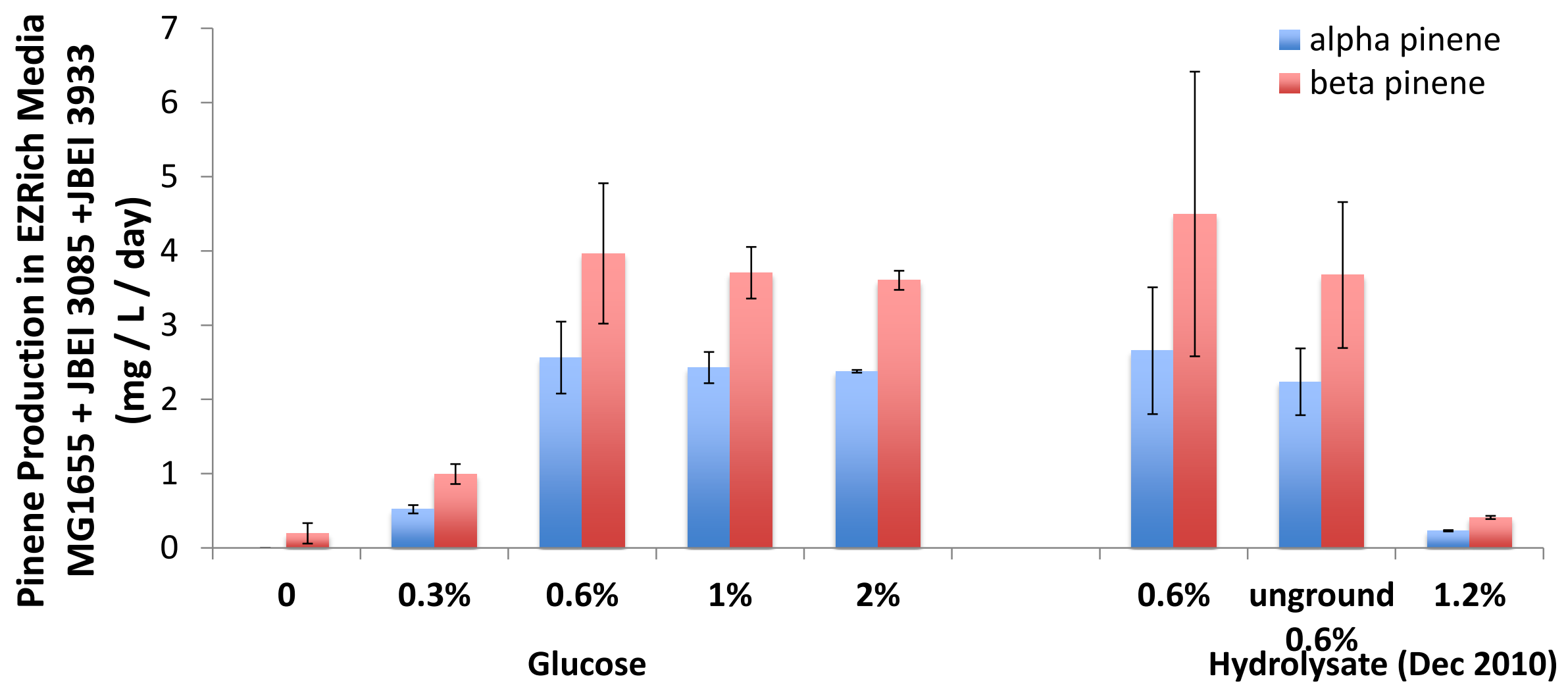

